\title{
Avances tecnológicos en la producción de alginatos en México
} Technological Advance for Alginate Production in Mexico

\author{
Hernández-Carmona G. \\ Centro Interdisciplinario de Ciencias Marinas \\ Instituto Politécnico Nacional \\ Correo:gcarmona@ipn.mx \\ Rodríguez-Montesinos Y.E. \\ Centro Interdisciplinario de Ciencias Marinas \\ Instituto Politécnico Nacional \\ Correo:yrodriguez@ipn.mx \\ Arvizu-Higuera D.L. \\ Centro Interdisciplinario de Ciencias Marinas \\ Instituto Politécnico Nacional \\ Correo:darvizu@ipn.mx
}

\author{
Reyes-Tisnado R. \\ Centro Regional de Investigaciones Pesqueras de La Paz \\ La Paz, Baja California Sur \\ Correo: rulreyes@prodigy.net.mx \\ Murillo-Álvarez J.I. \\ Centro Interdisciplinario de Ciencias Marinas \\ Instituto Politécnico Nacional \\ Correo:jmurilloa@ipn.mx \\ Muñoz-Ochoa M. \\ Centro Interdisciplinario de Ciencias Marinas \\ Instituto Politécnico Nacional \\ Correo:mmunozo@ipn.mx
}

Información del artículo: recibido: marzo de 2009, reevaluado: octubre de 2010, aceptado: mayo de 2011

\section{Resumen}

Los alginatos son polisacáridos que se extraen de las algas cafés. Estos tienen propiedades para formar geles y soluciones altamente viscosas, por lo que se emplean en la industria alimenticia, farmacéutica y textil, entre otras. Este trabajo de revisión describe las etapas optimizadas a nivel planta piloto para la producción de alginatos. El proceso consiste en tratar las algas con solución de $\mathrm{HCl}$ a pH 4, extraer el alginato con una solución de $\mathrm{Na}_{2} \mathrm{CO}_{3}$ a pH 10 calentando a $80^{\circ} \mathrm{C}$ y diluir la pasta y separar las partículas insolubles en un filtro rotatorio al vacío. El alginato en solución se precipita con una solución de $\mathrm{CaCl}_{2}$ para obtener fibras insolubles. Estas fibras se tratan con $\mathrm{HCl}$ para obtener el ácido algínico y finalmente se neutralizan con $\mathrm{Na}_{2} \mathrm{CO}_{3}$ para obtener alginato de sodio. El alginato se seca con aire caliente, se pulveriza y se tamiza a diferentes tamaños de malla. Se describen los diferentes productos obtenidos y sus propiedades físicas y químicas. Finalmente se resumen los costos de producción y se discuten las barreras que han limitado la producción de alginatos a nivel comercial en México, entre las que se incluyen la falta de un diseño industrial, el costo internacional de los alginatos, las políticas para otorgar las concesiones de los mantos de algas explotables y el papel de los inversionistas.

\section{Descriptores}

- algas

- alginatos

- costos

- polisacáridos

- producción 


\begin{abstract}
Alginates are polysaccharides extracted from brown seaweeds. They are used in food industry, pharmaceutical, textile, among other, because of their properties to give high viscous solution and gel forming. This review describes the optimized process at pilot plant level for alginate production. The process includes washing the algae with $\mathrm{HCl}$ at $\mathrm{pH} 4$, extraction of the alginate in $\mathrm{Na}_{2} \mathrm{CO}_{3}$ solution at $\mathrm{pH} 10$ and heating to $80^{\circ} \mathrm{C}$, dilution of the paste and filtration with a vacuum rotary filter. Alginate precipitation is carried out by adding $\mathrm{CaCl}_{2}$ filtration. The fibers obtained are treated with $\mathrm{HCl}$ to obtain alginic acid. The product is neutralized with $\mathrm{Na}_{2} \mathrm{CO}_{3}$ to obtain sodium alginate. The product is dried with hot air, milled, and screened at different mesh sizes. We described the different products obtained and their physical and chemical properties. Finally, costs and barriers found that limit the alginate production at commercial level in Mexico are discussed, including the lack of the industrial design, the international cost of the alginates, the policy to give the seaweeds beds concessions, and the role of the investors.
\end{abstract}

\section{Introducción}

Las algas marinas son recursos valiosos que se han utilizado de dos formas diferentes en la alimentación. Directamente en su forma natural (Nirmal et al., 2009) o bien, pueden ser procesadas para obtener compuestos químicos, que a su vez, se emplean en diversos tipos de industrias. A los compuestos extraídos de las algas que tienen la propiedad de formar soluciones viscosas o geles, se les llama ficocoloides. Los principales ficocoloides de importancia comercial son el alginato, que se extrae de las algas cafés; el agar y la carragenina que se extraen de las algas rojas (McHugh, 1987). Los alginatos son sales del ácido algínico que forman parte de la pared celular y de las regiones intercelulares de las feofitas. En su estado natural, se encuentran formando geles con iones $\mathrm{Ca}^{+2}, \mathrm{Na}^{+}, \mathrm{Mg}^{+2}, \mathrm{Sr}^{+2}$ y Ba ${ }^{+2}$ (Haug, 1964). Su función es conferir fuerza y flexibilidad al tejido del alga. El alginato es un polímero del ácido $\beta$-D-manurónico y el ácido $\alpha$-L-gulurónico, unidos por enlaces glucosídicos (1-4), agrupados en tres tipos de segmentos: D-manurónico $(\mathrm{M})$, L-gulurónico $(\mathrm{G})$ y un tercer segmento de diadas con ambos ácidos alternados (MM, GG, MG,). Estudios de resonancia magnética nuclear han demostrado que también hay secuencias de triadas (GGG, MGG, MGM) (Smidsrod y Draget, 1996; Murillo y Hernández, 2007; Yabur et al., 2007).

Los alginatos se utilizan ampliamente en la industria para darle consistencia y un aspecto adecuado a productos lácteos y productos enlatados; la textura es mejorada y la humedad es retenida con alginatos en productos de pastelería, como las mezclas para pasteles y los merengues. En alimentos congelados las propiedades de los alginatos aseguran la textura suave y el descongelamiento uniforme. La estabilización de la es- puma de la cerveza es una de las funciones más usuales de los alginatos. Para las impresiones en tela, se usan como espesante de la pasta que contiene la tinta. En la fabricación del papel produce una película suave y continua y una superficie con menos pelusa. Los alginatos también encuentran uso en la fabricación de varillas para soldar. En el campo de la medicina, el alginato se utiliza como agente desintegrador de tabletas y en la elaboración de vendas para heridas que son absorbidas por el cuerpo y no tienen que ser removidas (Hernández y Rodríguez, 1990). Los alginatos se usan para tratar úlceras gástricas, disminuyen el nivel de colesterol plasmático, inhiben las granulaciones y mastocitos, que están implicados en las reacciones de alergia (Nagaoka, et al., 2000). La elaboración de material de impresión dental (Reyes et al., 2004) y la inmovilización de organismos benéficos para el tratamiento de aguas residuales y como promotores del crecimiento de plantas (Yabur et al., 2007), son tecnologías que ya se encuentran estudiadas en México. Cada día se descubren más usos para estos compuestos en diferentes industrias y la mayoría de los estudios recientes sobre el tema de los alginatos se ha concentrado en nuevas aplicaciones como: acarreadores de proteínas (Coppi et al., 2001); intercambiadores de metales (Davis et al., 2004; De Stefano et al., 2005); incorporación en textiles (Gorenšek y Bukošek, 2006); inmovilización de levaduras (Pajic et al., 2007); micro encapsulados (Schuldt y Hunkeler, 2007; Mørc, 2008); absorción de radiación ultravioleta (Tavares, 2007); control de la colitis ulcerativa (Alireza et al., 2008), promotor del crecimiento de las raíces de las plantas terrestres (Iwasaki y Matsubara, 2000). Entre los usos indirectos, se encuentra que los residuos del proceso de extracción de alginatos, pueden ser utilizados como fertilizante, ya que la bacteria Gracilibacilus 
(A7) degrada el alginato a oligosacáridos durante el proceso de composta (Tang et al., 2009). Estudios recientes describen el potencial económico de la producción de bioetanol a partir de algas, basados en el hecho de que la materia prima puede derivarse de los residuos producidos por la industria de alginato, que está altamente enriquecida en azúcares como manitol y laminaran, de tal modo que los costos iniciales se reducen drásticamente (Moen et al., 1997; Horn et al., 2000). En el tema de producción sólo se han abordado las líneas generales del proceso (Draget et al., 2005; Mchugh, 2003) y la etapa de extracción (Vauchel et al., 2008a, 2008b y 2009).

Durante el año correspondiente al segundo semestre de 2007 al primer semestre del 2008, México importó 535 toneladas de alginatos de 12 países diferentes, entre los que destacan Estados Unidos, Francia, Reino Unido, Irlanda, Chile, Japón y Noruega. Un 80\% de la importación fue realizada por 17 empresas y se reporta un exportador, que probablemente revende lo que importa, ya que en México no hay fábricas de producción de alginatos. El costo de importación anual de alginatos fue de 6.2 millones de dólares (11.6 dólares por $\mathrm{kg}$ ). Los tipos de alginatos que se importaron fueron: $49.7 \%$ de alginato de propilenglicol (266 t a $\left.11.7 \mathrm{USD} \mathrm{kg}^{-1}\right), 45.3 \%$ de alginato de sodio $\left(242.5 \mathrm{t}\right.$ a $\left.10.2 \mathrm{USD} \mathrm{kg}^{-1}\right), 2.94 \%$ de alginato de magnesio, calcio y amonio (2.9 $\mathrm{t}$ a 29 USD $\left.\mathrm{kg}^{-1}\right), 1.44 \%$ de alginato de potasio $\left(7.7 \mathrm{t}\right.$ a $\left.12.8 \mathrm{USD} \mathrm{kg}^{-1}\right)$ y $0.5 \%$ de ácido algínico (2.8 t a $\left.19 \mathrm{USD} \mathrm{kg}^{-1}\right)$ (Secretaría de Economía, 2009).

La especie más importante para la producción de alginatos en México es el Sargazo Gigante (Macrocystis pyrifera) por su alto contenido de este ficocoloide y su abundancia (Hernández, 1985; Rodríguez y Hernández, 1991). Esta especie se distribuye en la costa noroccidental de la península de Baja California, desde la frontera con Estados Unidos hasta Punta San Hipólito, BCS. Se ha estimado que existe un potencial cosechable entre 35,812 t (invierno) a 97,803 t (verano) (en peso fresco) (Casas et al., 1985; Hernández et al., 1989a, 1989b, 1991). Esta especie tiene el ritmo de crecimiento más rápido que cualquier otro organismo marino. Cuando la temperatura es baja, existe una amplia disponibilidad de nutrientes y la cantidad de luz es abundante, sus frondas pueden llegan a crecer hasta $50 \mathrm{~cm}$ por día ${ }^{-1}$ (Reed, 1994). En México, su crecimiento promedio es $14.7 \mathrm{~cm} \mathrm{día}{ }^{-1}$ (verano) y $23.3 \mathrm{~cm} \mathrm{día}^{-1}$ (invierno). Esto sugiere que las algas podrían cosecharse dos veces al año con una regeneración de $70 \%$, sin que se presentaran efectos adversos para la planta o los organismos que viven asociados al Sargazo (Hernández, 1996). Macrocystis pyrifera se empezó a cosechar en 1958 con una producción promedio de 40,000 de toneladas anuales (peso fresco), la cual se vende a la compañía Kelco en California, EU para la extracción de alginatos; sin embargo, esta empresa cerró en 2006 y ahora se cosecha una pequeña porción para la elaboración de fertilizantes y como alimento para el abulón (Productos del Pacífico, com. pers.). Esta situación crea una oportunidad de inversión en la que se tiene una amplia disponibilidad de recurso sin explotar y además se cuenta con el proceso tecnológico para la obtención de alginatos.

El presente es un trabajo de revisión sobre lo que se ha desarrollado del tema de la producción de alginatos, a pesar de que ya se tienen algunas publicaciones científicas sobre las etapas independientes del proceso de producción (Hernández et al., 1989a, 1989b, 2002; McHugh et al., 2001), la comunidad científica e industrial no ha tenido acceso a la información de este proceso de forma completa y detallada, prueba de ello es que aún se publican trabajos en los que se emplean procesos menos eficientes o que son de uso en laboratorio y no son aplicables en la industria. De acuerdo con la revisión de la literatura, se ha encontrado que los procesos de extracción de alginatos que han utilizado otros autores, usan soluciones de reactivos que se preparan en ciertas concentraciones normales o porcentuales y se adicionan al proceso, por ejemplo: carbonato de sodio al 1\%. Esta forma de añadir los reactivos no considera el $\mathrm{pH}$ al que se llega en las diferentes etapas del proceso (Nishigawa, 1985; Younis et al., 2000; Fenoradosoa et al., 2010). Al no llevar las diferentes etapas al $\mathrm{pH}$ correcto de reacción, se puede ver afectado el rendimiento y la calidad del alginato obtenido, como se verá en las siguientes descripciones. Las etapas que se describen a continuación, contienen detalles que no habían sido publicados anteriormente.

\section{Proceso de producción de alginato de sodio a nivel planta piloto}

El fundamento de la extracción de alginato de las algas es convertir todas las sales de alginato en sales de sodio, disolver éstas en agua y eliminar los residuos insolubles por medio de una filtración. El proceso continúa con la adición de una sal de calcio para formar alginato de calcio, el cual tiene una textura fibrosa que no se disuelve en agua, y pueden ser separados del agua residual. El alginato de calcio se suspende en agua y se añade ácido para convertirlo en ácido algínico. Este ácido algínico fibroso se separa fácilmente. Después se coloca en un mezclador de tipo planetario con el alcohol y se añade poco a poco carbonato de sodio a la pasta, hasta que el ácido algínico se convierte en alginato de so- 
dio. La pasta de alginato de sodio se extrude en forma de pellets, que luego se secan y se muelen (McHugh, 2003).

\section{Reducción del tamaño de la materia prima}

Es necesario reducir el tamaño de las algas para favorecer las reacciones químicas del proceso y facilitar el transporte del material algal. Las algas pueden ser procesadas frescas o secas. La norma para el tamaño de las algas secas y molidas es la siguiente: $100 \%$ debajo de 6 $\mathrm{mm}$; $95 \%$ debajo de $3.3 \mathrm{~mm} ; 2 \%$ máximo debajo de 0.3 $\mathrm{mm}$. Las algas deben contener al menos $83 \%$ de materia seca (17\% de humedad) y menos del $3 \%$ de arena (Kelco, com. pers.). Para la molienda se emplea un molino de martillos convencional.

\section{Hidratación de las algas}

Para ablandar los tejidos y evitar la pigmentación del alginato, las algas se hidratan con una solución de formaldehído al 0.1\% (mínimo $15 \mathrm{~min}$, máximo $12 \mathrm{~h}$ ). Este tiempo depende de la especie. Algunas son más blandas que otras. Las especies con estipe grueso requieren hasta $12 \mathrm{~h}$. El formaldehído reacciona con los compuestos fenólicos que se encuentran dentro del alga para producir productos insolubles. La proporción de alga seca con respecto al agua es de 1:9. Esta proporción se debe minimizar a un nivel al que las algas absorban toda el agua posible y que al final de la hidratación no quede sobrenadante. Para el caso de Macrocystis pyrifera, a nivel planta piloto se procesaron $10 \mathrm{~kg}$ de alga con $90 \mathrm{~L}$ de solución de formaldehído al $0.1 \%$ con una pureza comercial de $37.5 \%$. Las algas se colocaron en un tanque de acero inoxidable de $87 \mathrm{~cm}$ de alto $\times 72 \mathrm{~cm}$ de diámetro. Se acopló al tanque un

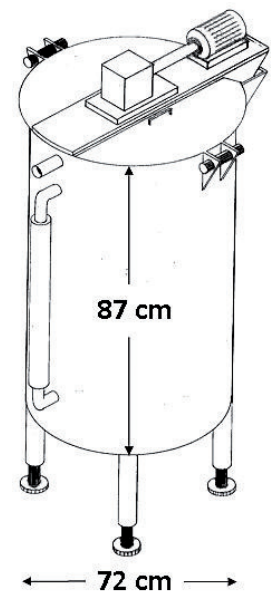

Figura 1. Tanque de hidratación y pre-extracción motor de $3 / 4$ de HP y un motorreductor con una velocidad de salida de $120 \mathrm{rpm}$. Se empleó un eje con aspas de tipo paleta inclinadas. Estas condiciones de proceso fueron suficientes para mover el volumen de agua y las algas durante el tratamiento (figura 1, foto 1A), (Hernández et al., 1999a).

$\mathrm{El}$ incremento en rendimiento de alginato, cuando se emplea el tratamiento con formaldheído, ha sido probado para algunas especies del género Sargassum (Davis et al., 2004)

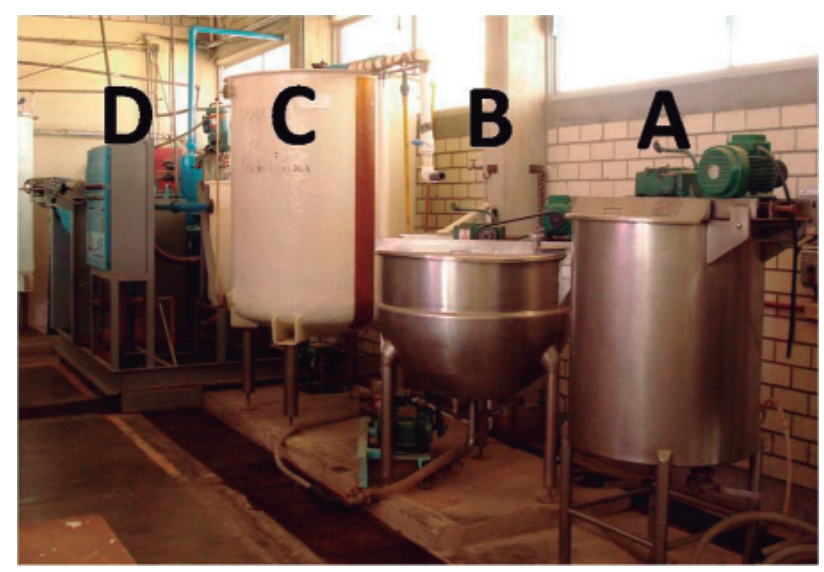

Foto 1. Plata piloto de producción de alginatos, A) tanque de hidratación y pre-extracción, B) marmita de exttracción, C) tanque de dilución, D) filtro rotatorio al vacío marca Alar

\section{Pre-extracción ácida}

Algunos autores mencionan que este tratamiento ácido se usa para convertir las sales de alginato de calcio y otros iones $\left(\mathrm{Na}^{+}, \mathrm{K}^{+}\right.$, etc.), en ácido algínico insoluble (Haug, 1964; Myklestad, 1968). Este intercambio no es necesario para la mayoría de las especies y el lavado sólo es para eliminar sales externas. Contrariamente, un tratamiento a $\mathrm{pH}$ menor a 4 , como proponen otros autores (Haug, 1964; Myklestad, 1968) puede producir depolimerización del alginato y bajar su viscosidad (Hernández et al., 1999a), así las algas hidratadas se drenan. La solución residual contiene laminaran y fucoidano, que se pueden separar por medio de precipitación con etanol. El drenado de las algas se puede llevar a cabo fácilmente si se tiene en el interior del tanque una malla. Las algas se dejan en el mismo tanque para el tratamiento con ácido clorhídrico (figura 1, foto 1A). Se adiciona agua al tanque con las algas hidratadas en una proporción de 10 partes de agua por una parte del alga seca inicial. Esta cantidad debe ser suficiente para que las algas se agiten libremente. Se agrega $\mathrm{HCl}$ industrial (con 24-28\% de pureza) hasta un pH de 4 en la solución. 
Las algas se agitan en el tanque por 15 min a temperatura ambiente y se drena la solución ácida residual. Las algas se lavan por 15 min con un volumen igual de agua (1:10) (McHugh, 1987; Hernández y Aguirre, 1987; Arvizu et al., 1995; Hernández et al., 1999a).

\section{Extracción}

Para extraer el alginato de la pared celular y la matriz intercelular de las algas, éstas se transportan por bombeo a un tanque enchaquetado con suministro de vapor. Para ello se empleó una marmita enchaquetada de acero inoxidable de $70 \mathrm{~cm}$ de altura máxima x $110 \mathrm{~cm}$ de diámetro, un motor de $3 / 4$ de HP y un motorreductor con una velocidad de salida de $120 \mathrm{rpm}$. Se empleó un aspa con tres paletas inclinadas, que fueron suficientes para mover eficientemente la pasta de alginato de sodio que se forma (figura 2, foto $1 \mathrm{~B}$ ). El alga pretratada se coloca en 16.6 partes de agua por una parte de algas (con base en el peso seco del alga inicial). Este volumen puede variar con la especie; las algas que producen baja viscosidad (ej. Sargassum) requieren menos agua (Rodríguez et al., 2008). Las algas que producen mayor viscosidad requieren más agua. Se calienta la solución a $80^{\circ} \mathrm{C}$ y se agrega suficiente carbonato de sodio en polvo para llegar a un pH 10. El volumen de agua debe ajustarse para formar una pasta que se pueda agitar, pero lo suficientemente espesa para que la fricción entre el líquido y las algas favorezca la extracción del alginato. Para estudiar esta etapa se diseñó un experimento en el que se midió la viscosidad de la solución a diferentes tiempos. La premisa era hacer la medición aún con las algas dentro de la solución (sin filtrar) a lo largo del tiempo de extracción para encontrar el máximo valor de viscosidad que se presenta en el proceso, ya que coincide con el máximo rendimiento posible. Esta viscosidad se denominó viscosidad de proceso o viscosidad aparente (Hernández et al., 1999b). Las mediciones de viscosidad aparente se realizaron en un viscosímetro Brookfield modelo LVT, con la aguja 4, que es la que se emplea para productos de alta viscosidad. Se sabe que las soluciones de alginato exhiben un comportamiento de carácter no-newtoniano, donde la viscosidad depende de la velocidad de deformación (shear rate), por lo que las mediciones se realizaron a la misma velocidad de rotación de la aguja $(60 \mathrm{rpm})$ para que estas fueran comparativas. Estas condiciones corresponden a una velocidad de corte o "shear rate" de $12.5 \mathrm{seg}^{-1}$. Esta forma de medición es la que comúnmente se emplea por la industria de los alginatos para evaluar su calidad en términos de viscosidad (Kelco, 1984), un valor final de viscosidad de proceso adecuado al final de la etapa es entre 3000 a $4000 \mathrm{mPa} \bullet \mathrm{s}$ y se logra después de 2 h de agitación. El pH de la solución puede disminuir a medida que el alginato se extrae del alga y se debe ajustar nuevamente a 10. En esta etapa se puede controlar la viscosidad del alginato a obtener. Las temperaturas elevadas y tiempos de agitación prolongados producirán alginatos de menor viscosidad, a diferencia de cuando se emplean menores temperaturas y tiempos de agitación, debido a la degradación del alginato por rompimiento de las cadenas de los ácidos urónicos que lo conforman. Es conveniente preparar una curva de variación de la viscosidad del alginato obtenido en función de los parámetros de tiempo y temperatura para cada especie a procesar, ya que las propiedades reológicas dependen de las condiciones de proceso, puesto que la cadena polimérica del alginato es especialmente sensible a temperaturas altas de extracción (Truss et al., 2001). Al final de la extracción el alga debe quedar prácticamente desintegrada (McHugh, 1987; Arvizu et al., 1996; Hernández et al., 1999b). El tiempo de extracción ( 2 h) también fue confirmado para Laminaria digitata (Vauchel et al., 2008b). Se ha propuesto una nueva alternativa para esta etapa llamada extrusión reactiva, en la que se reduce el consumo de agua y reactivo y se incrementa el rendimiento en 15\% (Vauchel et al., 2008a). Este grupo de investigadores franceses también desarrollaron un modelo que describe la cinética de esta etapa, en la que resalta el incremento del rendimiento a medida que se reduce el tamaño de partículas de algas (Vauchel et al., 2009). El incremento en rendimiento de alginatos a la temperatura de $80^{\circ} \mathrm{C}$ ha sido confirmado para especies del genero Sargassum (Davis et al., 2004).

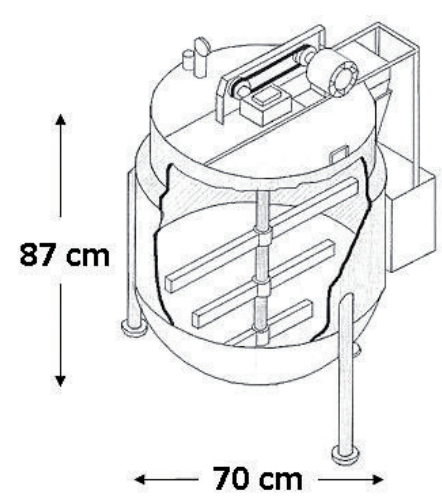

Figura 2. Marmita de extracción

\section{Dilución y filtración}

Después de la extracción, la pasta de alginato se bombea a un tanque con agitador y se combina con suficien- 
te agua para que la viscosidad se reduzca a $45 \mathrm{mPa} \bullet \mathrm{s}$. Se empleó un tanque de fibra de vidrio de $120 \mathrm{~cm}$ de diámetro x $150 \mathrm{~cm}$ de alto, con un serpentín en el interior. Se acopló al tanque un motor de $1 \mathrm{HP}$ y un agitador tipo propela, con velocidad de agitación de 1750 rpm (figura 3).

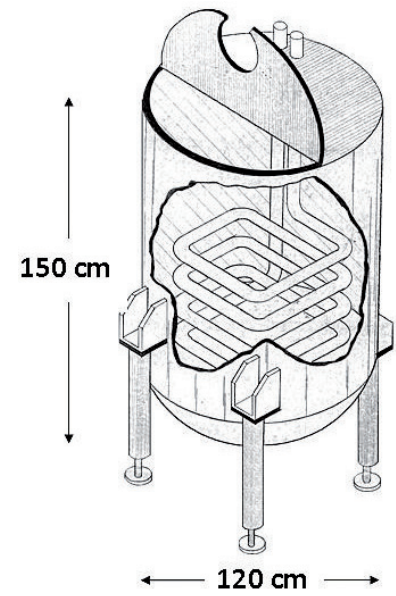

Figura 3. Tanque de dilución

El agua de lavado del tanque de extracción puede servir para bombear la pasta y diluir la solución a filtrar. El serpentín en el interior del tanque sirve para mantener la solución a una temperatura de $75^{\circ} \mathrm{C}$, lo que evita que aumente su viscosidad sin problemas de degradación. Para una especie como Macrocystis pyrifera, el volumen de agua para llegar a $45 \mathrm{mPa} \bullet \mathrm{s}$ y $75^{\circ} \mathrm{C}$ es de una parte de alga seca por 55 partes de agua.

Cuando la solución se ha ajustado a la viscosidad y temperatura mencionada, se bombea hacia un filtro rotatorio al vacío. Se puede emplear un equipo de la compañía ALAR (foto 2).

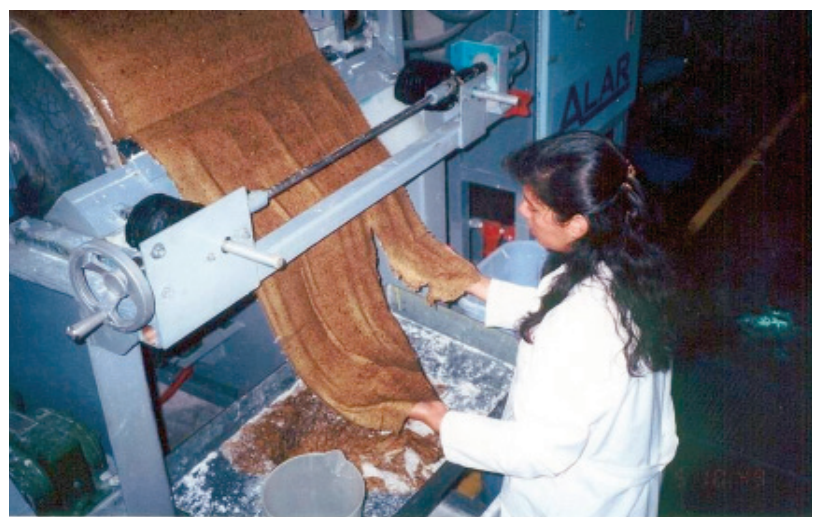

Foto 2. Filtro rotatorio al vacío empleado para la clarificación de la solución de alginato. Se observa el tambor giratorio y la capa de partículas de algas que se pegan a la cama de tierras de diatomeas por el efecto del vacío
El equipo se alimenta con la solución del tanque de dilución, bombeándola hacia la cuna receptora. El equipo cuenta al frente con una navaja que se mueve lentamente de manera automática a una velocidad de desplazamiento programada por el usuario, de manera que corta una pequeña cantidad del material filtrante a medida que el tambor está en rotación. A nivel planta piloto la navaja se mueve intermitentemente $1 \mathrm{~mm}$ cada 3.5 segundos y se mantiene una rotación del tambor de $2 \mathrm{rpm}$. El mejor material filtrante es la tierra de diatomeas (Celite 545). La lava expandida o perlita es la otra opción, la cual generalmente es más económica. Este dato es importante, porque se estima que se empleará 1 $\mathrm{kg}$ de material filtrante por $\mathrm{kg}$ de alginato producido (McHugh, 1987; Hernández et al., 1999b).

\section{Precipitación del alginato de calcio}

La solución de alginato sale a presión del filtro y llega a un tanque de precipitación a una temperatura de 50$60^{\circ} \mathrm{C}$. Al final de la tubería de descarga se instala un dispositivo tipo regadera para que la solución se descargue en el tanque de precipitación en forma de gotas. Se empleó un tanque de fibra de vidrio de $120 \mathrm{~cm}$ de diámetro $x 150 \mathrm{~cm}$ de alto, el cual fue equipado con un motor de $1 \mathrm{HP}$, con un reductor de velocidad variable y un aspa tipo propela para agitar la mezcla (figura 4). En un tanque independiente se prepara una solución de $\mathrm{CaCl}_{2}$ al $10 \%(\mathrm{p} / \mathrm{v})$. En la descarga también se tiene una salida en forma de regadera, de manera que la solución de $\mathrm{CaCl}_{2}$ también se descarga en forma de gotas. La cantidad total de reactivo a emplear corresponde a dos veces la cantidad de alginato a precipitar. Por ejemplo, si se va a precipitar una solución que contiene $2 \mathrm{~kg}$ de alginato, se emplearán $4 \mathrm{~kg}$ de $\mathrm{CaCl}_{2}$. La solución clarificada de alginato de sodio y la solución de $\mathrm{CaCl}_{2}$ se añaden a una velocidad sincronizada, de tal manera que ambas soluciones terminen de añadirse simultáneamente. Esta velocidad estará determinada por la velocidad de filtración. La velocidad de agitación debe ser controlada de tal manera que permita la formación de fibras pequeñas que puedan ser filtradas fácilmente. Si la agitación es muy débil, se formarán coágulos que son difíciles de filtrar. Si por el contrario, la agitación es muy fuerte, se formarán fibras muy pequeñas que serán difíciles de recuperar. La velocidad de agitación se debe acelerar a medida que el volumen en el tanque va aumentando, por eso es importante que el motor tenga velocidad variable. Es recomendable dejar las fibras dentro del tanque por lo menos $15 \mathrm{~min}$ antes de pasar a la siguiente etapa para que éstas terminen de reaccionar con el calcio y sea más fácil separarlas (McHugh, 1987; Hernán- 
dez y Casas, 1985; Arvizu et al., 1997; McHugh et al., 2001).

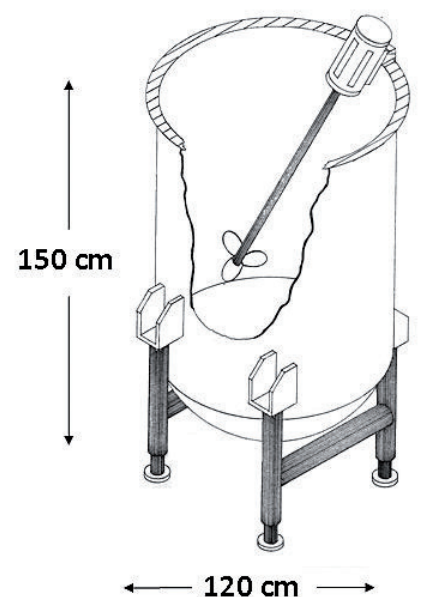

Figura 4. Tanque de precipitación

Conversión del alginato de calcio en ácido algínico

Las fibras de alginato de calcio se transforman en ácido algínico mediante lavados ácidos. Se pueden prensar, secar y moler para su venta como alginato de calcio, pero este producto tiene poco mercado, por lo que se transforma en ácido algínico u otra de sus sales. Las fibras de alginato de calcio se descargan por gravedad a una malla metálica (tipo mosquitero), si la precipitación se realizó bajo las condiciones adecuadas, el líquido podrá ser separado fácilmente. Las fibras no se deben comprimir para evitar interferencia en la siguiente reacción.

Se empleó un tanque de $100 \mathrm{~cm}$ de diámetro $\times 105$ $\mathrm{cm}$ de alto, con un motor de $1 \mathrm{HP}$ y un agitador con tres aspas tipo paleta (figura 5), Las fibras se depositan en el tanque y se adiciona suficiente agua para que se puedan agitar libremente, se adiciona $\mathrm{HCl}$ industrial hasta lograr un $\mathrm{pH}$ de 2 y se agita durante $15 \mathrm{~min}$. Las fibras se descargan sobre una malla, se llena nuevamente el tanque con agua, se regresan las fibras al tanque y se adiciona suficiente ácido para llegar a un $\mathrm{pH}$ de 1.8. Se agitan nuevamente por $15 \mathrm{~min}$ y se descargan las fibras. El procedimiento se repite para un tercer lavado con ácido a $\mathrm{pH}$ 1.8. A nivel industrial esta etapa se lleva a cabo en un sistema en contracorriente bajo el siguiente esquema, se usan tres tanques cuadrados que tienen un cuadro menor adicionado a un extremo, desde donde se suben las fibras a un transportador helicoidal. El alginato de calcio se agrega al primer tanque que contiene ácido previamente usado en el segundo tanque, después de agitar por $15 \mathrm{~min}$, el sólido (ahora una mezcla de ácido algínico y alginato de calcio) se transporta por medio de un gusano sin fin, que tiene una malla en el fondo, hacia el segundo tanque, el cual contiene ácido previamente usado en el tercer tanque. La agitación se repite y el sólido se alimenta al tercer tanque que contiene ácido sin usar, Después de agitar y separar el sólido, ahora como ácido algínico, se lava con agua; si ésta es abundante o si el agua es escasa, se manda sin lavar a la siguiente etapa. En esta etapa se puede controlar la viscosidad final del alginato, variando los $\mathrm{pHs}$ para limitar el intercambio iónico. Si se deja más calcio, se tendrá una mayor viscosidad aparente en el alginato, pero el límite de reducción de viscosidad del alginato en solución a 1\% (después de agregar hexametafosfato de sodio para secuestrar el calcio residual), debe ser menor a $40 \%$. Esta etapa se puede realizar lavando las fibras dos veces, solamente a $\mathrm{pH} 1.6$, o posiblemente a $\mathrm{pH} 1.8$, pero debe esperarse un poco de degradación del producto, con la consecuente reducción de viscosidad (Arvizu et al., 1997; Hernández et al., 2001; Rodríguez et al., 2005).

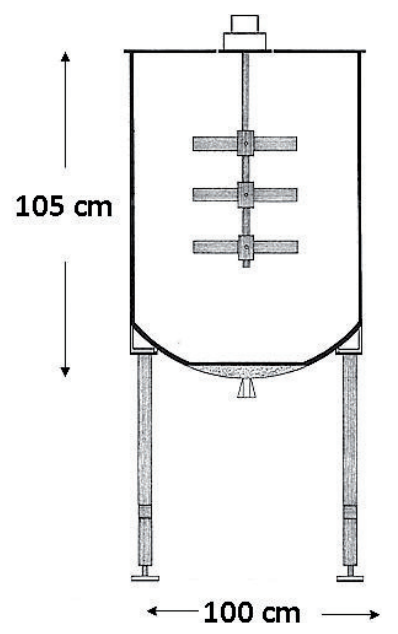

Figura 5. Tanque de lavados ácidos

\section{Prensado}

A nivel planta piloto, se empleó una prensa hidráulica con una canasta perforada. Las fibras se envuelven en una lona resistente y se someten a una presión de 8-10 toneladas. Se libera la presión, se revuelven las fibras y se prensan nuevamente. El proceso se repite hasta que ya no es posible eliminar más agua. A nivel industrial se puede emplear una prensa de tornillo o Screw Press, de la marca "Rietz S-Press" (http://www.bepex.com/spre ss. htm), en donde el líquido se elimina en tres áreas de la prensa para maximizar su salida y la recuperación del sólido. Está equipado con un motor independiente en el tornillo, que permite variar el tiempo de retención del 
producto. Este equipo permite reducir la humedad en las fibras hasta obtener un $25 \%$ de sólidos.

\section{Conversión del ácido algínico en alginato de sodio}

Esta es una de las etapas que más celosamente guardan las industrias. Las fibras prensadas de ácido algínico se colocan en un mezclador de doble planetario de 29 litros, con una compuerta para la adición de reactivos y otra para la toma de muestras (fibras de alginato) (foto 3); se agrega suficiente alcohol etílico al 96\%, de manera que la mezcla alcohol y agua, remanente en el ácido algínico, sea 55:45. Para la estimación de alcohol que se empleará durante la neutralización, se determina primero la cantidad de agua que se encuentra en las fibras de ácido algínico. Esto se obtiene restando el peso del ácido algínico húmedo menos el peso del alginato seco esperado, basado en un análisis previo a nivel laboratorio. Una opción adicional es determinar la humedad del ácido algínico.

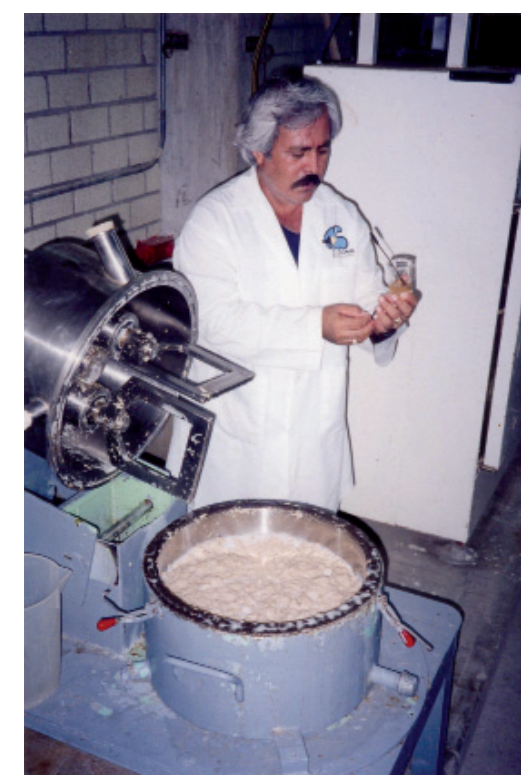

Foto 3. Mezclador de doble planetario para la conversión de ácido algínico en alginato de sodio

Ejemplificando lo anterior, si partimos de $23 \mathrm{~kg}$ de ácido algínico prensado (húmedo), que contiene $2.3 \mathrm{~kg}$ de alginato de sodio, se calcula que contiene $20.7 \mathrm{~L}$ de agua, que representa $55 \%$, por lo tanto, el volumen de alcohol para obtener una proporción de $45 \%$ es: $\mathrm{V}=$ $\left(45^{*} 20.7\right) / 55=16.9$ L de alcohol, mismo que será adicionado a las fibras. A continuación, se agrega una cantidad suficiente de $\mathrm{Na}_{2} \mathrm{CO}_{3}$ en polvo, hasta alcanzar un $\mathrm{pH}$ de 8 en las fibras después de agitar por $15 \mathrm{~min}$. Para medir el $\mathrm{pH}$ se toma una muestra de las fibras, se di- suelven en agua y se mide con papel indicador en la solución. La cantidad aproximada de $\mathrm{Na}_{2} \mathrm{CO}_{3}$ a emplear es de 0.25 partes por una de alginato de sodio a obtener. En esta etapa se puede sustituir el $\mathrm{Na}_{2} \mathrm{CO}_{3}$ por $\mathrm{K}_{2} \mathrm{CO}_{3} \mathrm{y}$ obtener alginato de potasio. El alcohol puede causar cierta interferencia en la medición del pH. Generalmente el $\mathrm{pH}$ del alginato en solución tendrá un grado menos del pH medido en la mezcla. La función del alcohol en esta etapa es evitar que cuando se convierta el ácido algínico en alginato de sodio, éste no se solubilice en el agua residual. Como el alcohol es caro, debe recuperarse en un destilador para reducir los costos; se estima que sólo $2 \%$ del alcohol se debe perder en la operación de recuperación. El alginato de sodio también se puede obtener sin el uso de alcohol, para lo cual se mezcla directamente con el carbonato de sodio, pero en este caso, se pierde la consistencia fibrosa y se tiene que introducir la pasta a un extrusor y obtener pelets, que posteriormente se pueden secar y pulverizar (McHugh, 1987; Arvizu et al., 2002; Hernández et al., 2002).

\section{Secado}

Para esta etapa se puede emplear un secador de aire caliente. Las fibras se prensan para eliminar los líquidos residuales, se desmenuzan y se colocan sobre charolas con bastidores de acero inoxidable y fondo de malla Núm. 40, que permite la circulación del aire. Con este sistema, la temperatura óptima de secado es de $60^{\circ} \mathrm{C}$ durante $2.5 \mathrm{~h}$, con lo que se obtiene una humedad en el alginato de $12 \%$ o menos. La máxima humedad recomendada para almacenar el alginato es de $12 \%$. Existen en el mercado secadores más eficientes. Lo más adecuado es enviar muestras del producto a los proveedores y determinar la cantidad de producto a procesar.

\section{Molienda, tamizado y mezclado}

Es necesario estandarizar el tamaño de partículas, de acuerdo a las especificaciones del mercado. El alginato de sodio seco se puede moler en un molino de turbina, también conocido como de martillos fijos. La mayoría de los alginatos requieren un tamaño de partícula menor a 60 mallas, lo cual se logra moliendo con una malla de $3 \mathrm{~mm}$. Para lograr productos de grano más fino se separa el polvo obtenido de la primera molienda y se vuelve a moler lo restante más grueso con una malla de $0.5 \mathrm{~mm}$. Es posible que algunos productos de tamaño muy fino requieran una tercera molienda. Para separar el alginato por tamaños se usa un tamizador con cinco tamices: $30(0.594 \mathrm{~mm}), 60(0.250 \mathrm{~mm}), 80(0.177 \mathrm{~mm})$, $100(0.149 \mathrm{~mm})$ y $120(0.125 \mathrm{~mm})$ mallas. Es difícil obte- 
ner partículas finas si el alginato es esponjoso, incluso después de tres moliendas se puede tener hasta un $16 \%$ de partículas que aún superan la malla 30 (Hernández et al., 2002). Existen en el mercado equipos muy sofisticados y eficientes que secan, muelen y clasifican en un solo aparato, pero son muy costosos.

El alginato obtenido se analiza en laboratorio para conocer la viscosidad del producto en solución al 1\%. La calidad de los alginatos (en términos de viscosidad) varía en función de diversos factores biológicos, ecológicos y de proceso. Por lo tanto, los productos que se obtengan tendrán una viscosidad diferente y deberán ser mezclados para ofrecer un producto de calidad constante. Para hacer las mezclas se usa un mezclador tipo pantalón y las viscosidades de los productos a combinar tienen que estar dentro del rango que se especifica entre paréntesis en $\mathrm{mPa} \bullet$ s: para 800 (> 600); 400 (150 a 600); 80 (40 a 150) y 30 (10 a 80). El producto final se empaca en cuñetes de cartón de $22.5 \mathrm{~kg}$ con una bolsa de plástico. La figura 6 muestra el diagrama de flujo para la producción de alginatos y las reacciones que están involucradas.

\section{Control de calidad}

A los alginatos obtenidos se les analizan las siguientes propiedades:

a) Viscosidad en solución al 1\% antes y después de agregar $0.5 \%$ de hexametafosfato de sodio, con relación al peso seco del alginato para secuestrar el calcio residual que queda ligado al alginato. La viscosidad se mide con un viscosímetro Brookfield LVT Modelo DV-I. Los productos se separan para su venta por su viscosidad de acuerdo con la siguiente clasificación medida en $\mathrm{mPa} \bullet \mathrm{s}$ : muy baja (25-35); baja (70-100); media (340-460) y alta (680-920).

b) $\mathrm{pH}$ de la solución a $1 \%$.

c) Distribución del tamaño de partículas, en donde los productos regulares se venden entre 30-60 mallas y los refinados entre 100-150 mallas.

d) Humedad, que debe ser menos de $12 \%$.

e) Cenizas, el estándar se encuentra entre $18-27 \%$.

f) Contenido de calcio, el estándar se encuentra entre $0.3-1 \%$.

g) Pureza, entre $96-98 \%$.

\section{Costos de producción}

La estimación de costos de producción se realizó considerando que una sola empresa cosechara todas las algas disponibles en la península (100,000 toneladas frescas) y que podrían obtener $25 \%$ de rendimiento promedio de alginato (base seca) (2,500 toneladas de alginato) con el proceso descrito. Los costos fueron calculados por kilo de alginato producido (datos en centavos de dólar), basados en la asesoría de un experto de la FAO, Com. Pers.). Como los datos no provienen de un análisis formal con una memoria de cálculo, deben ser considerados como una primera aproximación.

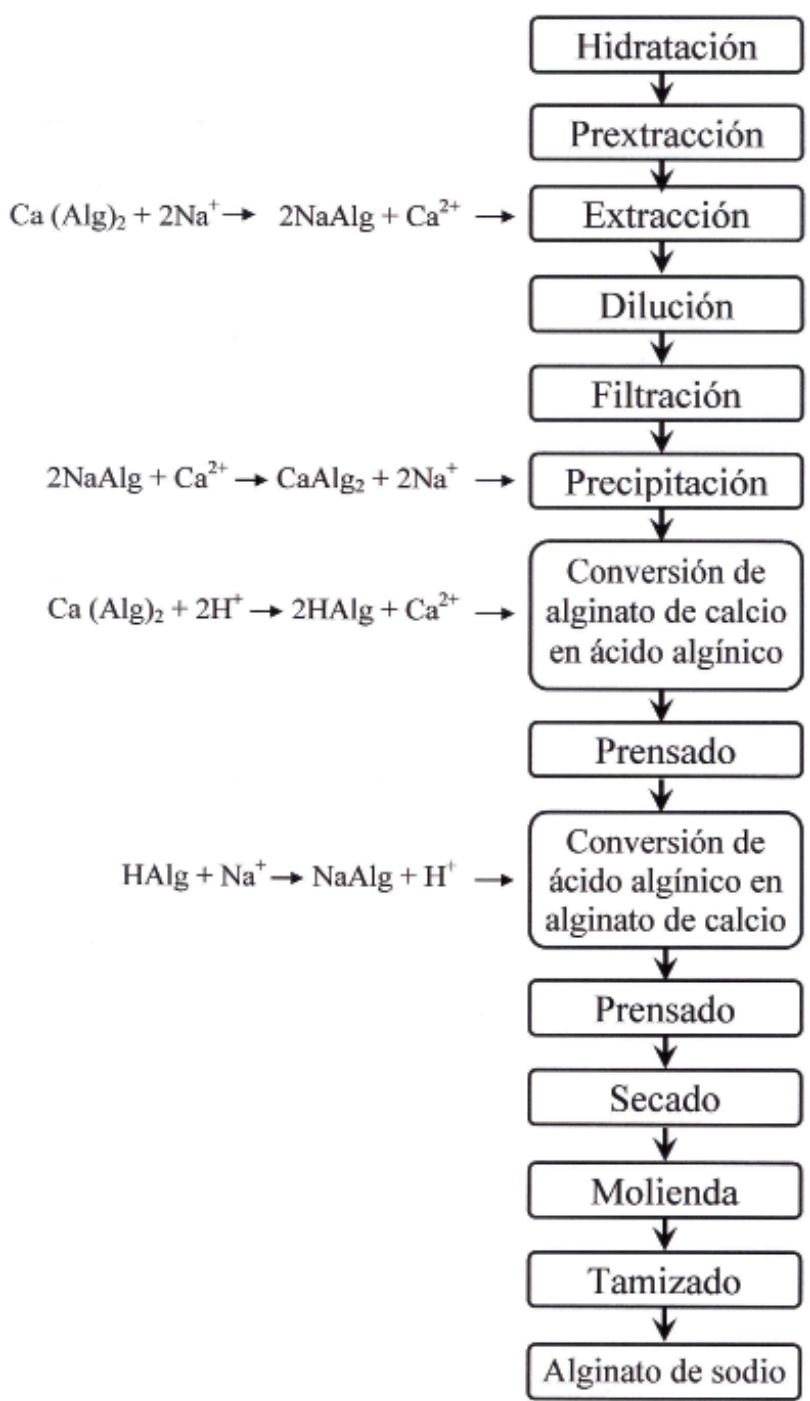

Figura 6. Diagrama de flujo del proceso de producción de alginatos 


\begin{tabular}{|c|c|}
\hline Concepto & $\begin{array}{c}\text { Centavos de } \\
\text { dólar Americano }\end{array}$ \\
\hline $\begin{array}{l}\text { Materias primas: considerando un barco tipo Sargacero como } \\
\text { el que operaba la empresa productos del Pacífico en Ensenada, } \\
\text { BC (Casas et al., 1996), más los reactivos (formaldehido, ácido } \\
\text { clorhídrico, carbonato de sodio, tierra de diatomeas, cloruro de } \\
\text { calcio, hipoclorito de sodio y etanol). }\end{array}$ & 194.44 \\
\hline Suministros: agua, electricidad y producción de vapor. & 40 \\
\hline $\begin{array}{l}\text { Mano de obra: para una fábrica en su mayor parte automatizada, } \\
\text { con seis obreros y un supervisor por turno y que opera en cuatro } \\
\text { turnos, en total } 14 \text { obreros y cuatro supervisores. }\end{array}$ & 15.36 \\
\hline $\begin{array}{l}\text { Mantenimiento: calculado en } 6 \% \text { de la inversión ( } 2.5 \text { millones de } \\
\text { dólares), } 50 \% \text { en materiales y } 50 \% \text { en mano de obra. }\end{array}$ & 24 \\
\hline Seguros e impuestos: se calculó en un $2 \%$ de la inversión inicial. & 8 \\
\hline Empaques: se calcularon 111,111 unidades de $22.5 \mathrm{~kg}$. & 2.2 \\
\hline $\begin{array}{l}\text { Ventas, gastos generales y administración; se calculó con base a } \\
15 \% \text { de los ingresos por ventas. }\end{array}$ & 135 \\
\hline Investigación y desarrollo: se consideró 5\% de las ventas. & 45 \\
\hline $\begin{array}{l}\text { Depreciación: se calculó sobre un tiempo de vida de los equipos } \\
\text { de } 11 \text { años (Baca, 1990). }\end{array}$ & 36 \\
\hline TOTAL & $\$ 5 \mathrm{USD}$ \\
\hline
\end{tabular}

Tabla 1. Costos de producción de alginatos en una fábrica instalada en México el que operaba la empresa productos del Pacífico en Ensenada BC (Casas et al., 1996), más los reactivos (formaldehido, ácido clorhídrico, carbonato de sodio, tierra de diatomeas, cloruro de calcio, hipoclorito de sodio y etanol).

Suministros: agua, electricidad y producción de vapor.

\section{Barreras identificadas para la producción industrial de alginatos}

A pesar de que en México existe la tecnología para la producción de alginatos, esta industria no se ha desarrollado, por lo que consideramos que está relacionado con los siguientes factores principales:

a) Esta tecnología no ha sido escalada a nivel industrial, por lo que se desconocen con precisión los costos de instalación.

b) Los mantos explotables se encuentran concesionados en dos regiones: $50 \%$ se encuentra en Baja California, aunque el nivel de explotación disminuyó, la compañía mantiene la concesión y el otro 50\% se encuentra concesionado a cinco cooperativas pesqueras del estado de Baja California Sur, pero ellos tampoco cosechan la biomasa disponible. En ambas regiones, se cosecha sólo una mínima parte que emplean como alimento para el cultivo de abulón.

c) Los costos de venta de los alginatos se han incrementado recientemente, actualmente el alginato de sodio se vende en promedio a 11.6 USD por kilo, por lo que si se produjera en México, la venta dejaría un margen de ganancia moderado (6.6 USD por kilo). El problema es convencer a los inversionistas de que ésta es una industria rentable. d) Otro factor son los inversionistas. Ellos quieren recibir las tecnologías desarrolladas sin costo y de tipo llave en mano, sin tener que invertir más en investigaciones previas al inicio de la producción. Consideramos que esta actitud debe cambiar, para que las tecnologías desarrolladas en las instituciones lleguen a aplicarse a nivel industrial.

e) Un último factor es que el proceso de producción de alginatos consume grandes cantidades de agua dulce $\left(1000 \mathrm{~m}^{3}\right.$ por tonelada de producto final), por lo que la ubicación de la planta deberá pensarse en un lugar con abundante agua dulce y aplicar los procesos de recirculación que se han propuesto (Hernández et al., 1992; Reyes et al., 1992; Rodríguez et al., 2005).

En conclusión, en México existe la tecnología para la producción de alginatos y se encuentra disponible para su uso de manera gratuita, ya que las bases científicas del proceso han sido publicadas en beneficio de la comunidad internacional. La siguiente etapa que se debe abordar es el diseño industrial. Éste debe estar lleno de ingenio, de manera que nos permita reducir al máximo los costos de producción y producir con alta calidad. También es conveniente involucrar a contadores que apliquen sus conocimientos en la búsqueda de una producción rentable. Con esta información, será factible 
hacer una campaña para fomentar la explotación de las algas entre los concesionarios, invitándolos a que unifiquen esfuerzos y ofreciéndoles la asesoría que requieren para la instalación de esta industria.

\section{Agradecimientos}

Los autores agradecen las becas otorgadas por exclusividad a la COFAA-IPN y el Estímulo al Desempeño de los investigadores del IPN. Agradecemos a la FAO, Instituto Politécnico Nacional, CONACYT y SEMARNAT, los apoyos económicos recibidos para el desarrollo de este proyecto.

\section{Referencias}

Alireza-Razavi A., Khodadadi A., Bagher-Eslami M., Eshraghi S., Mirshafiey A. Therapeutic Effect of Sodium Alginate in Experimental Chronic Ulcerative Colitis. Iranian Journal of Allergy, Asthma and Immunolgy, 7(1):13-18, marzo, 2008.

Arvizu-Higuera D.L., Hernández-Carmona G., Rodríguez-Montesinos Y.E. Sistemas de carga y de flujo continuo durante la etapa de preextracción ácida en el proceso de extracción de alginatos. Ciencias Marinas, 21(1):25-37, marzo 1995.

Arvizu-Higuera D.L., Hernández-Carmona G., Rodríguez-Montesinos Y.E. Efecto de la temperatura y el tiempo de extracción en el proceso de extracción de alginato de sodio a partir de Macrocystis Pyrifera. Ciencias Marinas, 22(4):511-521, diciembre 1996.

Arvizu-Higuera D.L., Hernández-Carmona G., Rodríguez-Montesinos Y.E. Efecto del tipo de precipitación en el proceso de obtención de alginato de sodio: método de alginato de calcio y método de ácido algínico. Ciencias Marinas, 23(2):195-207, junio 1997.

Arvizu-Higuera D.L., Hernández-Carmona G., Rodríguez-Montesinos Y.E. Parámetros que afectan la conversión de ácido algínico a alginato de sodio. Ciencias Marinas, 28(1):27-36, marzo 2002.

Baca-Urbina G. Evaluación de proyectos: análisis y administración de riesgo, McGraw Hill, México, 1990.

Casas-Valdez M.M., Hernández-Carmona G., Torres-Villegas J.R., Sánchez-Rodríguez I. Evaluación de mantos de Macrocystis Pyrifera "Sargazo Gigante" en la Península de Baja California (Verano de 1982). Investigaciones Marinas CICIMAR, 2(1):1-17, marzo 1985.

Casas-Valdez M., Hernández-Carmona G., Hernández-Guerrero C.J. Recurso Macrocystis Pyrifera, en: Casas-Valdez M., PonceDíaz G. (eds.), Estudio Potencial Pesquero y Acuícola de Baja California Sur II. Secretaría del Medio Ambiente Recursos Naturales y Pesca, Baja California Sur, México, 1996. pp. 431-444.

Coppi G., Iannuccelli V., Leo E., Bernabei M.T., Cameroni R. Chitosan-Alginate Microparticles as a Protein Carrier. Drug Development and Industrial Pharmacy, 27(5):393-400, enero 2001.
Davis T.A., Ramirez M., Mucci A., Larsen B. Extraction, Isolation and Cadmium Binding of Alginate From Sargassum spp. Journal of Applied Phycology, 16(4):275-284, agosto 2004.

De Stefano C., Gianguzza A., Piazzese D., Sammartano S. Modelling of Proton and Metal Exchange in the Alginate Biopolymer. Analytical and Bioanalytical Chemistry, 383(4):587-596, octubre 2005.

Draget K.I., Smidsrod O., Skjak-Braek G. Alginates from Algae, en: Steinbüchel A., Rhee S.K. (eds.), Polysaccharides and Polyamides in the food Industry: Properties, Production and Patents I. Wiley-VCH, Weinheim, 2005, pp. 1-30.

Fenoradosoa T.A., Ali G., Delattre C., Laroche C., Petit E., Wadouachi A., Michaud P. Extraction and Characterization of an Alginate from the Brown Seaweed Sargassum Turbinarioides Grunow. J Appl Phycol, 22:131-137, mayo 2010.

Gorenšek M., Bukošek V. Zinc and Alginate for Multipurpose Textiles. Acta Chimica Slovenica, 53(2):223-228. Diciembre 2006.

Haug A. Composition and Properties of Alginates. Report 30. Norwegian Institute Seaweed Ressearch, Thondheim, Noruega, 1964, pp 123.

Hernández-Carmona G., Casas-Valdez M.M. Precipitación del ácido algínico y su conversión a alginato de sodio en muestras de Macrocystis Pyrifera. Investigaciones Marinas. CICIMAR, 2(1):18-28, marzo 1985.

Hernández-Carmona G. Variación estacional del contenido de alginatos en tres especies de feofitas de Baja California Sur, México. Investigaciones Marinas. CICIMAR, 2(1):29-45, marzo 1985.

Hernández-Carmona G., Aguirre-Vilchis M. Propiedades de intercambio iónico de Macrocystis Pyrifera durante la pre-extracción ácida, para la extracción de alginatos. Investigaciones Marinas. CICIMAR, 3(2):53-64, 1987.

Hernández-Carmona G., Rodríguez-Montesinos Y.E., Torres-Villegas J.R., Sánchez-Rodríguez I., Vilchis M.A. Evaluación de los mantos de Macrocystis Pyrifera (Phaeophyta, Laminariales) en Baja California, México, I. Invierno 1985-1986. Ciencias Marinas, 15(2):1-27, junio 1989a.

Hernández-Carmona G., Rodríguez-Montesinos Y.E., Torres-Villegas J.R., Sánchez-Rodríguez I., Vilchis M.A., García-de-LaRosa O. Evaluación de los mantos de Macrocystis Pyrifera (Phaeophyta Laminariales) en Baja California, México. II Primavera 1986. Ciencias Marinas, 15(4):117-140, 1989b.

Hernández-Carmona G., Rodríguez-Montesinos Y.E. Los alginatos: Un producto útil a partir de algas marinas. CICIMAR Boletín 4:1-1, 1990.

Hernández-Carmona G., Rodríguez-Montesinos Y.E., Casas-Valdez M.M., Vilchis M.A., Sánchez-Rodríguez I. Evaluación de los mantos de Macrocystis Pyrifera (Phaeophyta, Laminariales) en la península de Baja California Sur, México. III. Verano de 1986 y variación estacional. Ciencias Marinas, 17(4):121-145, diciembre 1991.

Hernández-Carmona G., Vilchis M.A., Rodríguez-Montesinos Y.E. Recirculación del ácido residual de la etapa de pre-extrac- 
ción en el proceso de obtención de alginato de sodio. Ciencias Marinas, 18(1):125-137, marzo 1992.

Hernández-Carmona G. Tasas de elongación de frondas de Macrocystis pyrifera en Bahía Tortugas, Baja California. Ciencias Marinas, 22(1):57-72, febrero 1996.

Hernández-Carmona G., McHugh D.J., Arvizu-Higuera D.L., Rodríguez-Montesinos Y.E. Pilot Plant Scale Extraction of Alginate from Macrocystis Pyrifera. Part 1. The Effect of Pre Extraction Treatments on the Yield and Quality of Alginate. Journal of Applied. Phycology, 10(6):507-513, diciembre 1999a.

Hernández-Carmona G., McHugh D.J., López-Gutiérrez F. Pilot Plant Scale Extraction of Alginates from Macrocystis Pyrifera. 2. Studies on Extraction Conditions and Methods of Separating the Alkaline-Insoluble Residue. Journal of Applied Phycology, 11(6):493-502, diciembre 1999b.

Hernández-Carmona G., Robledo D., Serviere-Zaragoza E. Effect of Nutrient Availability on Macrocystis Pyrifera Recruitment Survival Near its Southern Limit of Baja California. Botanica Marina, 44(3):221-229, junio 2001.

Hernández-Carmona G., McHugh D.J., Arvizu-Higuera D.L., Rodríguez-Montesinos Y.E. Pilot Plant Scale Extraction of Alginates from Macrocystis Pyrifera 4. Conversion of Alginic Acid to Sodium Alginate, Drying and Milling. Journal of Applied Phycology, 14(6):445-451, diciembre 2002.

Horn S.J., Aasen I.M., Østgaard K. Ethanol Production from Seaweed Extract. Journal of Industrial Microbiology and Biotechnology, 25: 249-254, noviembre 2000.

Iwasaki K., Matsubara Y. Purification of Alginate Oligosaccharides with Root Growth-Promoting Activity Toward Lettuce. Biosci Biotechnol Biochem., 64: 1067-1070, mayo 2000.

Kelco. Algin/Hydrophilic Derivatives of Alginic Acid for Scientific Water Control. 50 pp. 1984.

McHugh D.J. Production and Uutilization of Products from Commercial Seaweeds, FAO Fisheries Technical Paper 288, Roma, 1987, pp. 58-115.

McHugh D.J., Hernández-Carmona G., Arvizu-Higuera D.L., Rodríguez Montesinos Y.E. Pilot Plant Scale Extraction of Alginates from Macrocystis Pyrifera. 3. Precipitation, Bleaching and Conversion of Calcium Alginate to Aalginic Acid. Journal of Applied Phycology, 13(6):471-479, diciembre 2001.

McHugh D.J. A Guide to the Seaweed Industry, FAO Fisheries Technical Paper, Núm.441, Rome, FAO, 2003, 105 pp.

Moen E., Horn S., Ostgaard K. Alginate Degradation During Anaerobic Digestion of Laminaria Hyperborea Stipes. Journal of Applied Phycology, 9: 157-166, mayo 1997.

Mørc Ý.A. Novel Alginate Microcapsules for Cell Therapy. Tesis (Ph. D.), Norwegian University of Science and Technology, 2008, Pp. 74.

Murillo-Álvarez J.I., Hernández-Carmona G. Monomer Composition and Sequence of Sodium Alginate Extracted at Pilot Plant Scales from three Commercially Important Seaweeds from México. Journal of Applied Phycology, 19(5):545-548, octubre 2007.
Myklestad S. Ion-Exchange of Brown Algae. Determination of Rate Mechanism for Calcium Hydrogen Ion Exchange for Particles from Laminaria hyperborea and Laminaria digitata. Journal of Applied Chemistry, 18:30-36, 1968.

Nagaoka M., Shibata H., Kimura-Takagi I., Hashimoto S. AntiUlcer Effects and Biological Activities of Polysaccharides from Marine Algae. BioFactors, 12: 267-274, enero 2000.

Nirmal-Kumar J.I., Kumar R.N., Patell K., Viyol S., Bhoi R. Nutrient Composition and Calorific Value of Some Seaweeds from Bet Dwarka, West Coast of Gujarat, India. Our Nature, 7:18-25, mayo 2009.

Nishigawa K. Extract Method of Alginic Acid, en: Research Methods of Algae. Nishigawa K., Chthara M., 1985, pp. 624-626.

Pajic-Lijakovic I., Plavsic M., Nedovic V., Bugarski B. Investigation of Ca-Alginate Hhydrogel Rheological Behavior in Conjunction with Immobilized Yeast Cell Growth Dynamics. Journal of Microencapsulation, 24(5):420-429, agosto 2007.

Reyes-Tisnado R., López-Gutiérrez F., Hernández-Carmona G., Vernon-Carter J., Castro Moroyoqui P. Sodium and Potassium Alginates Extracted from Macrocystis Pyrifera Algae for Use in Dental Impression Materials. Ciencias Marinas, 30(3):189-199, 2004.

Reyes-Tisnado R., Hernández-Carmona G., Rodríguez-Montesinos Y.E., Arvizu-Higuera, D.L. Food Grade Alginates Extracted from the Giant Kelp Macrocystis Pyrifera at Pilot Plant Scale. Revista de Investigaciones Marinas, 26:185-192, 2005.

Rodríguez-Montesinos Y.E., Hernández-Carmona G. Variación estacional y geográfica de la composición química de Macrocystis Pyrifera en la Costa Occidental de Baja California. Ciencias Marinas, 17(3):91-107, septiembre 1991.

Rodríguez-Montesinos Y.E., Hernández-Carmona G., Arvizu-Higuera D.L. Aprovechamiento de los líquidos residuales en la etapa de conversión de alginato de calcio en ácido algínico durante el proceso de producción de alginatos. Oceánides, 20(1,2): 1-7, diciembre 2005.

Rodríguez-Montesinos Y.E., Arvizu-Higuera D.L., HernándezCarmona G. Seasonal Variation on Size and Chemical Constituents of Sargassum Sinicola Setchel et Gardner from Bahía de La Paz, Baja California Sur, México. Phycological Research, 56(1): 34-39, marzo 2008.

Schuldt U., Hunkeler D. Alginate-Cellulose Sulphate-Oligocation Microcapsules: Optimization of Mass Transport and Mechanical Properties. Journal of Microencaption, 24(1):1-10, marzo 2007.

Secretaría de Economía, Negociaciones comerciales [en línea]. 2009. Disponible en: http://www.economia-snci.gob.mx.

Smidsrod O., Draget K.I. Chemistry and Physical Properties of Alginates. Carbohydrate in Europe, 14:6-13, mayo 1996.

Tang J.C., Taniguchi H., Chu H., Zhou Q., Nagata S. Isolation and Characterization of Alginate Degrading Bacteria for Disposal of Seaweeds Waste. Letters in Applied Microbiology, 48:38-43, enero 2009. DOI: 10.1111/j.1472-765X.2008.02481.x. 
Tavares-Salgado L, Tomazetto R, Paes-Cinelli L, Farina M, Menezes-Amado-Filho G. The Influence of Brown Algae Alginates on Phenolic Compounds Capability of Ultraviolet Radiation Absorption in Vitro. Brazilian Journal of Oceanography, 55(2):145 154, abril-junio 2007.

Truus K., Vaher M., Taure I. Algal Biomass from Fucus Vesiculosus (Phaeophyta): Investigation of the Mineral and Alginate Components. Proc. Estonian Acad. Sci. Chem., 50(2):95-103, junio 2001.

Vauchel P., Arhaliass A., Legrand J., Kaas R., Baron R. Decrease in Dynamic Viscosity and Average Molecular Weight of Alginate from Laminaria digitata During Alkaline Extraction. Journal of Phycology, 44(2):515-517, abril 2008a.

Vauchel P., Kaas R., Arhaliass A., Baron R., Legrand J. A New Process for Extracting Alginates from Laminaria Digitata: Reactive Extrusion. Food and Bioprocess Technology, 1(3):297-300, septiembre 2008b.

Vauchel P., Leroux K., Kaas R., Arhaliass A., Baron R., Legrand J. Kinetics Modeling of Alginate Alkaline Extraction from Laminaria Digitata. Bioresource Technology, 100(3):1291-1296, febrero 2009.

Yabur R., Bashan Y., Hernández-Carmona G. Alginate from the Macroalgae Sargassum Sinicola as a Novel Source for Microbial Immobilization Material in Wastewater Treatment and Plant Growth Promotion. Journal of Applied Phycology, 19(1):43-53, febrero 2007.

Younis Y.M.H., Tecleab S., Gheza T., Rahama I.H. Determination of the Biomass and Phycocolloid Contents of some Marine Algae from Eritrea. J. KAU: Mar. Sci., 11:19-25, 2000.

\section{Bibliografía}

Edwards M.S., Hernández-Carmona G. Delayed Recovery of Giant Kelp Near its Southern Range Limit in the North Pacific Following “El Niño". Marine Biology, 147(1):273-279, mayo 2005.

Hernández-Carmona G., García O., Robledo D., Foster M. Restoration Techniques for Macrocystis Pyrifera Populations at the Southern Limit of their Distribution in Mexico. Botanica Marina, 43(3):273-284, junio 2000.

Hernández-Carmona G., Hughes B., Graham M. Reproductive Longevity of Drifting Kelp Macrocystis pyrifera (Phaeophyceae) in Monterey Bay. Journal of Phycology, 42(6):1199 1207, diciembre 2006.

Istini S., Ohno, Kusunose M. Methods of Analysis of Agar, Carrageenan and Alginates in Seaweeds. Bull. Mar. Sci. Fish. Kochi Univ., 14:49-55, diciembre 1994.

Lada L., Zertuche-González J.A., Hernández-Carmona G. Giant kelp (Macrocystis pyrifera, Phaeophyceae) Recruitment near its Southern Limit in Baja California After Mass Disappearance During ENSO 1997-98. Journal of Phycology, 35(6):1106-1112, diciembre 1999.

Reed D.C. Giant Forest of the Sea. Natural Science, pp. 202-207, 1994. Reyes-Tisnado R., Hernández-Carmona G., HernándezValenzuela R. Reducción del consumo de agua dulce en el proceso de extracción de alginatos a partir de Macrocystis Pyrifera (Phaeophyta, Laminariales), mediante recirculación de los líquidos residuales de la pre-extracción y precipitado. Ciencias Marinas, 18: 105-124. 1992. 


\section{Semblanza de los autores}

Gustavo Hernández-Carmona. Biólogo marino por el CICIMAR-IPN, doctorado en ciencias por el Centro de Investigaciones Biológicas del Noroeste. Realizó una estancia de investigación en los laboratorios marinos de Moss Landing, California. Profesor-investigador de tiempo completo del CICIMAR. Ha dirigido 5 tesis de licenciatura, 13 de maestría y 3 de doctorado. Ha publicado 39 artículos científicos en revistas internacionales, 4 memorias de congresos, 3 capítulos de libros y 13 artículos de divulgación. Ha participado con 44 ponencias en congresos nacionales y 45 internacionales. Su investigación está orientada a la química y ecología de las macroalgas, con énfasis en la producción de ficocoloides y uso de las macroalgas y sus productos.

Yoloxochitl Elizabeth Rodríguez-Montesinos. Se graduó como bióloga marina en la Universidad Autónoma de Baja California Sur, México. Obtuvo la maestría en ciencias marinas en el Centro Interdisciplinario de Ciencias Marinas-IPN, México. Es profesor desde 1990. Su línea de investigación es la química de algas marinas. Ha participado en proyectos encaminados al estudio de extracción de polisacáridos algales de interés comercial y en el diseño de una planta piloto para la extracción de alginatos, así como el estudio de polisacáridos con actividad biológica. Ha dirigido una tesis de maestría y publicado 19 artículos científicos y 2 de divulgación. Ha participado en 25 congresos nacionales e internacionales.

Dora Luz Arvizu-Higuera. Es ingeniero bioquímico egresada del Instituto Tecnológico de La Paz; realizó su maestría en ciencias marinas en el Centro Interdisciplinario de Ciencias Marinas del IPN. Cuenta con 11 publicaciones científicas internacionales, en revistas indexadas. Labora en el CICIMAR-IPN desde 1994, en el área de química de macroalgas, con experiencia en la extracción de polisacáridos algales. Actualmente es profesor-investigador con categoría titular C. Ha participado en diversos proyectos de investigación sobre alginatos, agar y búsqueda de compuestos bioactivos.

Raúl Reyes-Tisnado. Ingeniero bioquímico en ciencias marítimas y tecnología de alimentos del Instituto Tecnológico y de Estudios Superiores de Monterrey. Doctorado en ciencias marinas del CICIMAR-IPN. Laboró como profesor de tiempo completo en el ITESM-Mazatlán. Desde 1985 a la fecha es investigador titular en el Instituto Nacional de Pesca. Su perfil profesional incluye las áreas de química y extracción de ficocoloides, legislación pesquera, economía pesquera, biología pesquera, biología marina, métodos de pesca, acuacultura, oceanografía, contaminación marina, biotecnología marina, ingeniería y procesado de alimentos, administración de recursos humanos, negociación y manejo de conflictos, administración de recursos marinos, formulación e implementación de proyectos de investigación de desarrollo tecnológico marino-pesquero.

Jesús Iván Murillo-Álvarez. Ingeniero bioquímico en alimentos por el Instituto Tecnológico de La Paz. Doctorado en ciencias por el Centro de Investigaciones Biológicas del Noroeste. Ha realizado estancias de investigación en Copenhague y USA. Es profesor titular del CICIMAR-IPN. Ha escrito 10 artículos científicos en revistas internacionales. Ha participado en 6 congresos nacionales y 11 congresos internacionales. Es miembro de la American Society of Pharmacognosy, la Sociedad de Ficología de Latinoamérica y el Caribe. Es miembro del Sistema Nacional de Investigadores, Nivel I. Su investigación está orientada al estudio de los organismos marinos como fuente de sustancias activas.

Mauricio Muñoz-Ochoa. Ingeniero bioquímico por el Instituto Tecnológico de la Paz. Doctor en ciencias por el Centro Interdisciplinario de Ciencias Marinas del IPN. Es profesor asociado del CICIMAR-IPN. Ha participado con 6 ponencias en congresos nacionales y 13 ponencias en congresos internacionales. Ha participado en 9 proyectos de investigación. La línea de investigación que realiza está enfocada al aislamiento y purificación de sustancias bioactivas a partir de organismos marinos. 